\title{
TAIL: Two-level Approach for Indoor Localization
}

\author{
V. Sudha Rani and S. V. Raghavan \\ Network Systems Laboratory \\ Department of Computer Science and Engineering \\ Indian Institute of Technology Madras, Chennai - 600 036, INDIA \\ \{sudharani, svr\}@cs.iitm.ernet.in
}

\begin{abstract}
Popularity of ubiquitous computing increases the importance of location-aware applications, which increases the need for finding location of the user. In this paper, we present a novel localization method for indoor environments using $\mathrm{Wi}-\mathrm{Fi}$ infrastructure.

While localization using Wi-Fi is cost effective, handling the obstructions which are the main cause of signal propagation error in indoor environments is a challenging task. We address this problem in two levels, resulting in increased accuracy of localization. In the first level, we "localize" the residing area of user node in coarse granularity. Then, we use building layout to find the objects that attenuate the signal between the reference node and the coarse estimate of the location of user node. Using multi-wall propagation model, we apply corrections for all obstructions and find the location of user node. Empirical results based on experiments conducted in lab-scale, shows meter-level accuracy.
\end{abstract}

Keywords: Propagation Model, Ubiquitous Computing, Wi-Fi

\section{Introduction}

Use of wireless technology has become pervasive in our day-to-day life. Besides, a wide range of applications make use of location information to improve performance or to enhance user convenience. Localization is the process of finding out the location of a node - either by itself or by a central server. The most popular localization technology Global Positioning system (GPS) [10] is best suited for outdoors. Practical limitations such as cost, power, precision, and inaccessibility render the choice of using GPS for positioning in indoor environments infeasible. Indoor localization techniques have been proposed hitherto, differ either in cost of deployment or in accuracy or both. Among these, the localization techniques which use Wi-Fi technologies based on $802.11 \mathrm{x}$ standards obviates the need for installing special hardware. Moreover these are found almost everywhere - in buildings, in organizations, in public utility services and in common usage areas such as hospitals, airports, and restaurants. In the sequel, we propose an approach using $802.11 \mathrm{x}$ based equipment that provides meter-level accuracy which seems to be sufficient for most emerging applications. 
The indoor localization techniques proposed thus far can be classified into two classes viz; range-based and range-free techniques. Range-based techniques rely on range measurements to estimate the distance to the reference nodes, using Infrared or Ultrasound or $802.11 \mathrm{x}$ technology and then uses trilateration or multilateration technique, to calculate the exact position of user node. On the contrary, range-free techniques use only the connectivity information from $802.11 \mathrm{x}$ reference nodes to estimate the location. Range-based techniques require either installation of additional hardware like Infrared or Ultrasound receivers or can lead to calibration overhead, where as range-free techniques require dense deployment of reference nodes to achieve appreciable accuracy.

The major source of error for localization using $802.11 \mathrm{x}$ infrastructure in indoor environments is the obstructions that interfere with the signal propagation from the reference node to user node. The attenuation caused is dependent on the number and type of obstructions. The accuracy of the localization method can be increased if these obstructions are identified and necessary corrections are applied to the signal strength during distance estimation. But the actual number and type of obstructions between user node and the reference node depends on the exact location of user node, which is normally unknown. To overcome this problem, a Two-level approach is proposed in this paper. The two levels in the proposed approach are - Macro and Micro. In Macro level, the Locality ${ }^{l}$ of user node using range-free technique is computed. Then the number and type of obstructions are found using the computed Locality and knowledge of the building layout. In Micro level, corrections to the signal strength are applied using the obstructions found, which are then fed to range-based technique to find the location of user node.

Depending on the applications, the accuracy required varies from coarse-grain accuracy to fine-grain accuracy. For example, finding the nearest printer in an office requires coarse-grain accuracy, where as finding the exact position of the patients in emergency conditions in a hospital requires fine-grain accuracy. The technique proposed in this work can adapt to these varying application requirements. We achieve this by adjusting the cell size; large cells for coarse-grain requirements and small cells for fine-grain requirements.

\section{Related Work}

As explained in previous section, work in the area of indoor localization of a node can be classified broadly into two categories, viz; range-based and range-free techniques. Cricket [1], Active Badge [2] and APS [3] fall into range-based category. The common principle behind the range-based techniques is to find the distance between user node and the reference node using either time of flight or received signal strength (RSS) as the measure. Having found the distances to a minimum of three reference nodes, multilateration technique is applied to calculate the location of user

\footnotetext{
${ }^{1}$ Portion of a building where user is residing
} 
node. The techniques which take time of flight as a metric, require installation of additional hardware either at user node or at the reference node. Even though RSS technique doesn't require installation of additional hardware, finding distance to the reference nodes based on received signal strength is not accurate in indoor environments due to the attenuation caused by obstructions. To overcome the difficulty of predicting signal propagation, radio map technique RADAR [4] has been proposed which works in two phases - offline and online. In offline phase the strength of the signals from the reference nodes are measured and stored in the database at all positions of the operating environment and in online phase the received signal strengths are compared with the ones stored in the database to find the location of user node. The disadvantage of this method is that tedious work is called for during offline phase and the process has to be repeated if the position of even one reference node changes which is not infrequent in real world. Besides, maintaining the database is an issue when operating in large buildings.

Proposals based on range-free techniques are described in [5], [6], [7] and [9]. All these techniques use radio connectivity information of the reference nodes in finding the location. These techniques overcome the calibration costs encountered in RSS and offline work involved in the RADAR. Besides, these techniques obviate the need for additional hardware. But, the accuracy of range-free techniques is less compared to RSS-based techniques, as it uses only connectivity information. Appreciable accuracy of range-free localization techniques can be achieved only when the density of reference nodes is more or when reference nodes are distributed uniformly, or when the number of neighboring user nodes is more [9]. Satisfying these conditions at all times cannot normally be guaranteed in practical situations.

The work reported in this paper overcomes all the above limitations and still gives appreciable accuracy. We demonstrate through experiments that the error-in-estimation in the proposed technique is less than two meters.

\section{Wireless Channel Characteristics}

The basic property of the radio propagation is that, the received signal strength value decreases as the distance between transmitter and receiver increases which makes it a suitable metric for relative-distance measurement. The distance to signal-strength relation is captured in signal propagation model. Most widely used propagation model for indoor environments is log distance path loss model [8], which is given as:

$$
R S S(d)=P t-P L\left(d_{0}\right)-10 \eta \log _{10}\left(d / d_{0}\right)
$$

In this equation $R S S(d)$ is the strength of the signal received from the reference node at distance of $d$ meters, $P_{t}$ is the transmitted power, $P L\left(d_{0}\right)$ is the path loss for a reference distance of $d_{0}$ [8], and $\eta$ is the path loss exponent. While usage of this propagation model to predict the signal strength is simple, it doesn't take into account 
the operating environment which impacts radio propagation. Therefore, propagation model captured in equation (1) is best suited for obstruction free environments but not for indoor environments where obstructions are abound.

To consider the effect of obstructions on the signal strength, multi-wall propagation model [8] is used in the proposed work. The building layout data is used to identify the obstructions that are present in the path between transmitter and receiver. This in turn can be used to make necessary corrections to the received signal strength, based on the number and type of obstructions present between the transmitter and receiver. The generalized propagation model can then be given as:

$$
R S S(d)=P_{t}-P L\left(d_{0}\right)-T L(d)
$$

where $T L(d)$ represents the total transmission loss for a particular distance $d$ which is composed of two components: the path transmission loss $(P L(d))$ and the loss due to obstructions $(O L)$. Hence the total transmission loss can be given as:

$$
T L(d)=P L(d)+O L
$$

Obstruction Loss $(O L)$ depends on the number of obstructions between the transmitter and the receiver and is sum of attenuations which is given as:

$$
O L=\sum_{i=1}^{n} m_{i} x_{i}
$$

where $m_{i}$ is the number of obstructions of a particular type $i$ and $x_{i}$ is the attenuation caused by it. OL drops down to zero when line of sight conditions exist between transmitter and receiver and increases with increase in the number of obstructions. Therefore multi wall propagation model is given as:

$$
R S S(d)=P-P L\left(d_{0}\right)-10 \eta \log _{10}\left(d / d_{0}\right)-\sum_{i=1}^{n} m_{i} x_{i}
$$

The parameter $x_{i}$ for each type of obstruction was determined empirically from a series of experiments conducted under controlled conditions. Next, we will explain the experimental setup followed by the results obtained.

We conducted two experiments, one to show that log normal propagation model holds good when there is line of sight between sender and receiver and the second one to understand the effect of obstruction on the signal from sender to receiver. The 
experiments were called Line Of sight (LOS) Propagation Model experiment and Non-LOS propagation Model experiment respectively.
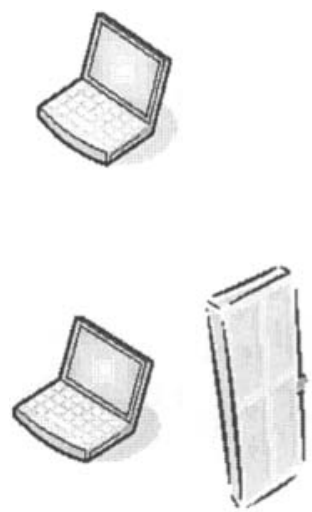

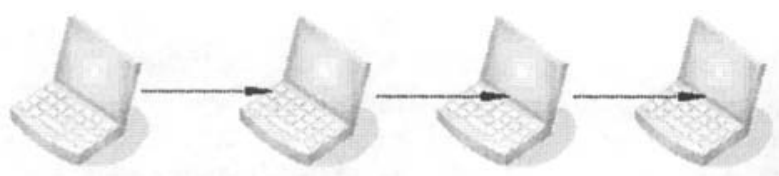

Fig. 1. LOS Experiment setup

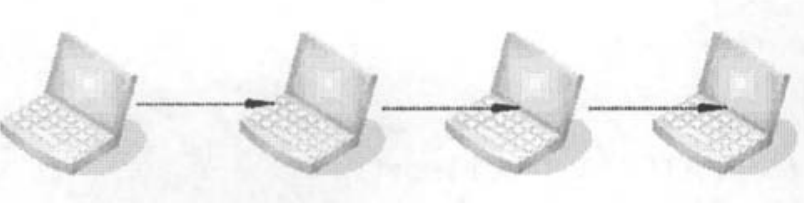

Fig. 2. NLOS Experiment setup

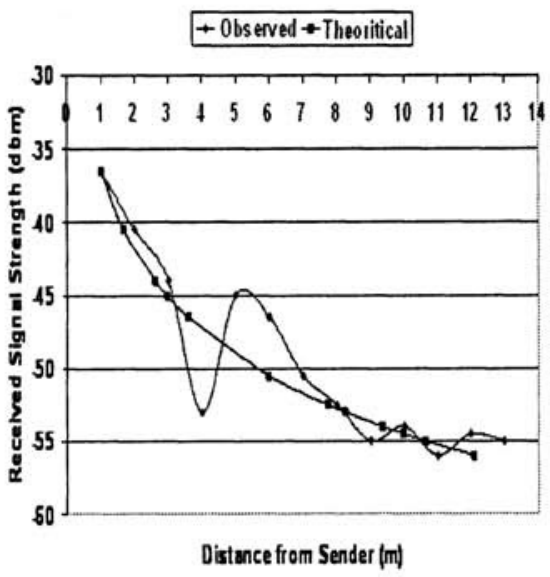

Fig. 3. LOS Propagation Model

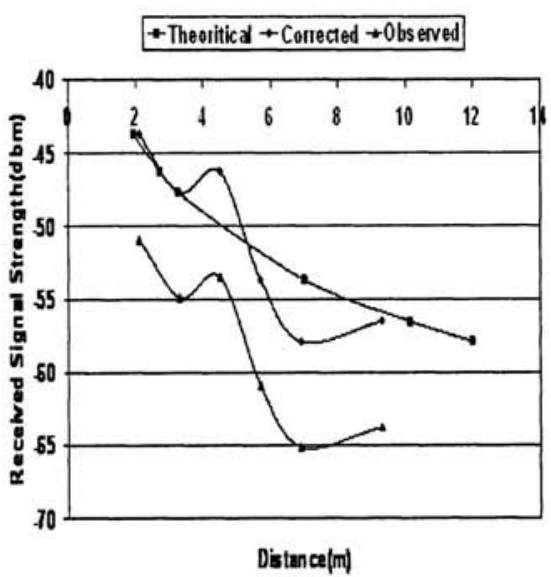

Fig. 4. NLOS Propagation Model

The experimental setup for these two experiments is shown in Fig. 1 and Fig. 2 respectively. Two laptops were taken, of which one was the reference node and the other was user node. NetStumbler software was installed in user node which captured the signal strength of the broadcast messages sent by the reference node. The signal strength readings were measured at various distances from the reference node. In LOS experimental setup, Line of Sight condition exists between the reference node and user node, where as in NLOS setup, an obstruction was kept between the reference node and user node to find the attenuation loss of this obstruction. 
We observe from Fig. 3 that, in LOS experiment the theoretical and observed values correlate each other. In the NLOS case, we observe (Fig. 4) that the observed values shifted and are aligned with theoretical values after the correction was applied. The hump in these figures can be attributed to the random noise in the environment.

However, since the number and type of obstructions cannot be known apriori, globally accepted propagation model is difficult to obtain. The actual number of walls between user node and the reference node depends on the exact location of user node, information that is normally unknown. The naive approach to estimate the position is to find the signal strength vector at various points using multi wall propagation model [8] and compare it with the obtained one. The downside of this approach is large processing time, when the building size is more. To overcome this, we propose a two-level approach to find the location of user node.

\section{TAIL: System Model}

In this work, we consider the localization system consisting of a set of $802.11 \mathrm{~b}$ reference nodes and a set of user nodes located on a 2-dimensional floor-plan. Each reference node sends broadcast messages that contain the location information and the unique Id periodically. User nodes measure the signal strength from each reference node from these broadcast messages and Id is used to differentiate between different reference nodes. Using this information along with the priori knowledge of the building layout and the locations of the reference nodes, the position of user node is determined. The reference nodes are randomly distributed in the given floor-plan. Even though optimal placement of reference nodes increases the accuracy of the proposed method, it is not examined in this work.

\section{TAIL: $\underline{T}$ wo-level $\underline{A} p p r o a c h$ for $\underline{\text { Indoor Localization }}$}

As mentioned earlier in section 1, the major hurdle for localization algorithm is ranging errors which occur due to obstructions that attenuate the signal. To overcome these errors, the proposed technique works in two levels namely Macro level and Micro level. Range-free and range-based techniques have been applied in these levels respectively. Macro level estimates the Locality of user node, the size of which depends on the number of reference nodes in range. Micro level takes this Locality information as input, computes number and type of obstructions, and finally uses corrected multi-wall propagation model (equation 5) to compute the location of user node. The following subsections explain each level in detail.

The proposed technique requires the storage of only the building layout and the attenuation loss of the obstructions present in the building, making it suitable to implement either in a centralized manner - where a central server finds the location of all user nodes, or in a distributed manner - where user node finds its own location. It is assumed that network level connectivity exists between user node and central server 
and the underlying communication technology is $802.11 \mathrm{x}$. The choice between centralized and distributed approaches can be made depending on the requirements of the application, power constraints of user nodes and privacy issues involved. Distributed implementation requires, every user node to be provided with building layout and the attenuation values of the obstructions. In a centralized implementation, central server stores this information and either user node transmits the received signal strength readings to the server, or the reference nodes measures the signal strength of the messages from user node and sends them to the central server.

\subsection{Macro Level}

Macro level applies range-free technique to estimate the Locality of user node. Range-free technique uses the overlapping coverage region of reference nodes to estimate the required area [7]. The reference nodes having IDs $-\mathrm{ID}_{1}$ to $\mathrm{ID}_{\mathrm{n}}$ are situated at positions $\left(X_{l}, Y_{l}\right)$ to $\left(X_{n}, Y_{n}\right)$ with transmission ranges $R_{l}$ to $R_{n}$ respectively. These reference nodes broadcast messages periodically which contain their ID and location coordinates. User node listens for these broadcast messages for a period of time and lists the reference nodes which it is able to hear. User node can receive the broadcast messages of the reference node if and only if it is in the coverage region of the reference node, which is the circle drawn with the reference node location as the center and its transmission range as the radius. If user node is able to hear from more than one reference node, then the overlapping coverage region of all these reference nodes correspond to the Locality of user node. Here it has to be observed that the signal strength of the messages from the reference node is not being taken into consideration, so the accuracy of the returned Locality will not be affected by the attenuation error caused by the obstructions.

This is shown pictorially in Fig. 5, where A1, A2 and A3 are the reference nodes and user node is receiving broadcast messages from all the three nodes. The Locality of user node is shown as shaded region in Fig. 5. Increase in the number of reference nodes in its range, results in finer granularity of the region. In the following paragraph, we present the Cell Table approach which returns the Locality as the set of cells.

The whole operating environment is divided into cells as shown in the Fig. 6, where each square represents one cell. Each cell is assigned an integer called cell rank which is set to zero initially for all the cells. This cell rank represents the number of reference nodes in its range. Each cell is checked for the validation of In-Range property detailed below to decide if the cell is included in the transmission range of the reference node. If the cell satisfies this property, the rank of the cell is incremented by one otherwise the value remains unchanged. This process is repeated for all the reference nodes from which user node has received the broadcast messages. 


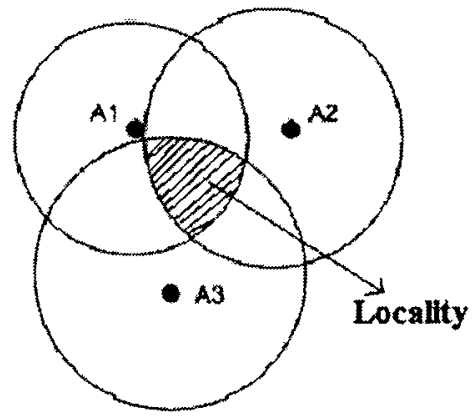

Fig. 5. Intersection Approach

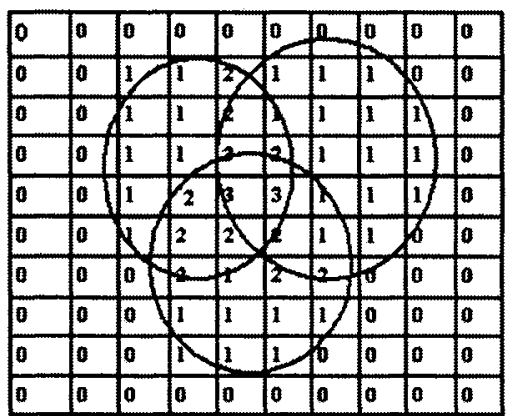

Fig. 6. Cell Table

The In-Range property checks whether the cell is in the coverage region of the corresponding reference node or not, which can be formulated as below:

$$
P:\left(x_{c}-x_{i}\right)^{2}+\left(y_{c}-y_{i}\right)^{2} \ll R_{i}^{2}
$$

In this equation, $\left(x_{c}, y_{c}\right)$ are the coordinates of the center of cell, $\left(x_{i}, y_{i}\right)$ are the coordinates of one of the reference nodes from which the node has received broadcast messages and $R_{i}$ is its transmission range. The property is satisfied when the distance between the center of cell and the reference node is less than its transmission range.

The overlapping region i.e. the Locality of user node is defined as the set of cells which are having the highest rank in the cell table. In the Fig. 6, user node is in the range of three reference nodes, so the Locality corresponds to the set of these cells whose rank is three. The pseudo code for Cell Ranking is as follows:

For each cell $\mathrm{C}$ of the building

For each reference node $\mathrm{ID}_{\mathrm{i}}$ user node is hearing

$$
\begin{aligned}
& \text { if }\left(\left(\mathrm{x}_{\mathrm{c}}-\mathrm{x}_{\mathrm{i}}\right)^{2}+\left(\mathrm{y}_{\mathrm{c}}-\mathrm{y}_{\mathrm{i}}\right)^{2}-\mathrm{R}_{\mathrm{i}}^{2}<=0\right) \\
& \text { then increment cell rank by } 1 ;
\end{aligned}
$$

\}

\subsection{Micro Level}

Micro level estimates the cell in which user node is residing from the set of cells obtained in Macro level. As said previously, most of the error in indoor environments is due to the attenuation caused by obstructions between the transmitter and the receiver. If the number of obstructions between the reference node and user node were 
known, then the distance to the reference node can be determined using the multi-wall propagation model given in equation (5) by applying the correction as explained in section 3 . However, the actual number of obstructions between the reference node and user node depends on the exact location of user node and is thus unknown. To circumvent this problem, for all the cells obtained in the Macro level, signal strength vector is estimated by finding the obstructions and using equation (5), which is then compared with the received signal strength vector. The location of user node is defined as the center of the cell whose estimated signal strength vector best matches with the received signal strength vector. This is explained in detail with the help of an example in the following paragraph.

Consider the layout plan shown in Fig. 7, where reference nodes are shown as A1, A2 and $\mathrm{A} 3$ and square $\mathrm{C}$ represents one of the cells obtained from the Macro level. The obstructions between cell $\mathrm{C}$ and each of the reference nodes can be determined as the positions of cell, reference nodes and layout plan is known. For example, in the Fig. 7 shown, the number of obstructions from the cell center to the reference nodes A1, A2 and $\mathrm{A} 3$ are 1, 3 and 3 respectively. Taking this information into consideration signal strength vector is estimated using equation (5).

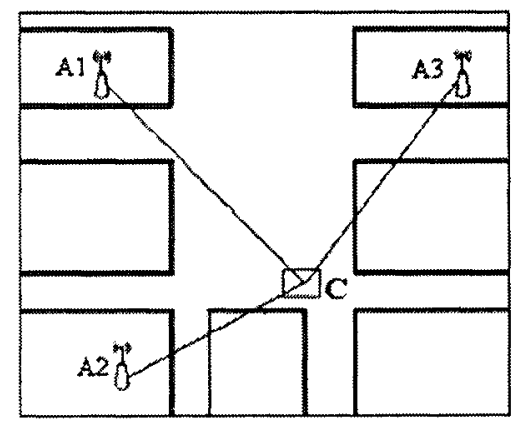

Fig. 7. Building Layout

This signal strength vector is estimated for each cell obtained from the Macro level. It is of the form $\mathrm{S}_{\mathrm{i}}=\left(\mathrm{s}_{\mathrm{i} 1}, \mathrm{~s}_{\mathrm{i} 2}, \mathrm{~S}_{\mathrm{i} 3} \ldots . . \mathrm{s}_{\mathrm{ik}}\right)$, for all $i=1$ to $N$, where $N$ is number of cells obtained in Macro level and $\mathrm{k}$ is the number of reference nodes covering the obtained cells and $s_{i j}$ denotes the signal strength at cell i from the $j^{\text {th }}$ reference node. The received signal strength vector is represented as $\mathrm{R}=\left(\mathrm{r}_{1}, \mathrm{r}_{2}, \mathrm{r}_{3} \ldots . \mathrm{r}_{\mathrm{k}}\right)$. The Euclidean distance given in equation (7) is calculated between the received signal strength vector and the estimated signal strength vector for all the cells.

$$
D\left(S_{i}, R\right)=\sqrt{\sum_{j=1}^{k}\left(S_{i j}-r_{j}\right)^{2}}
$$




$$
\text { CellNumber }=\min _{i}\left(D\left(S_{i}, R\right)\right) \text {. }
$$

The CellNumber with minimum Euclidean distance is found as shown in equation (8), whose center is taken as the location of user node.

Extension of the proposed method to 3-dimensional environment is straight forward by taking $\mathrm{z}$-coordinate in equation (6) and taking attenuation loss due to floors between transmitter and receiver into account in multi wall propagation model given in equation (5).

\section{TAIL: Experimental Results}

We conducted a series of experiments under controlled conditions to evaluate the performance of TAIL. All the experiments are conducted in two-dimensions by keeping all the reference nodes and user node at the same height. Initially the attenuation loss of all the obstructions present in the environment were calculated as explained in section 3. Though this is time consuming, it is a one-time process and these values can be used even if the underlying 802.11 infrastructure changes.

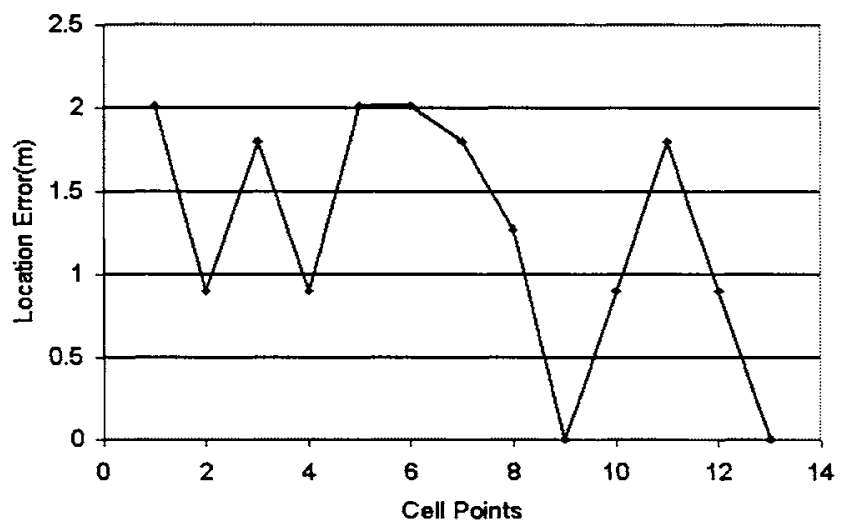

Fig. 8. Location Estimation Error for various points

The considered infrastructure contains four reference nodes and one user node. The reference nodes were static. Netstumbler software package was installed in user node to capture the signal strength of the messages sent by the reference nodes. The building was divided into cells of size $0.9 \times 0.9 \mathrm{~m}$. User moved along various cells and measured both the actual position and signal strength readings from the reference nodes. The location of user node was estimated with these signal strength vectors using the proposed two-level approach. The location estimation error was calculated 
as the distance between the actual location and estimated location. Let $(x, y)$ represent the actual location and $\left(x_{e s t}, y_{e s}\right)$ represent the estimated location. The error is given by

$$
\text { Error }=\sqrt{\left(x_{e s t}-x\right)^{2}+\left(y_{e s t}-y\right)^{2}}
$$

The graph in Fig. 8 shows the location error for various points. The $x$-axis shows various points in the building and the $y$-axis shows error in meters. It can be observed from Fig. 8 that the error of the proposed technique is less than 2 meter for all the cells.

\section{TAIL: Simulation Results}

We have done simulation in Matlab to see the effect of number of reference nodes and size of cell on location accuracy. Size of the building was taken as $60 \times 30 \mathrm{~m}^{2}$ and parameter values of the multi-wall propagation model used are given in Table 1 . A random noise with $\sigma^{2}=4 \mathrm{~dB}$ was added to the model and positions of the reference nodes and user nodes were selected randomly inside the building.

Table 1. Parameter Values for Propagation Model

\begin{tabular}{|l|l|}
\hline$d_{o}$ & 1 meter \\
\hline$P_{t}-P L\left(d_{0}\right)$ & $-38 \mathrm{dBm}$ \\
\hline$\eta$ & 2.5 \\
\hline$R$ & 30 meters \\
\hline$x_{i}$ & $4 \mathrm{~dB}$ \\
\hline
\end{tabular}

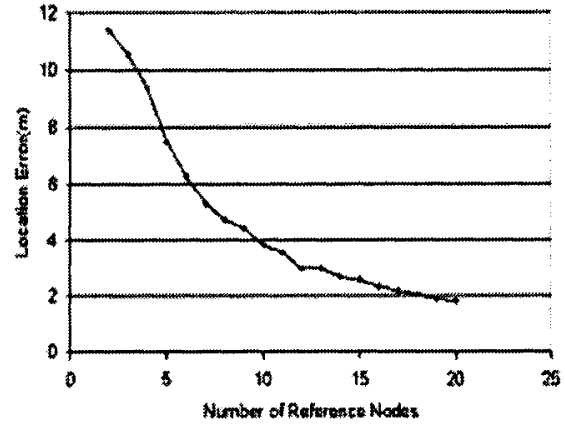

Fig. 9. Location Error against number of Reference Nodes

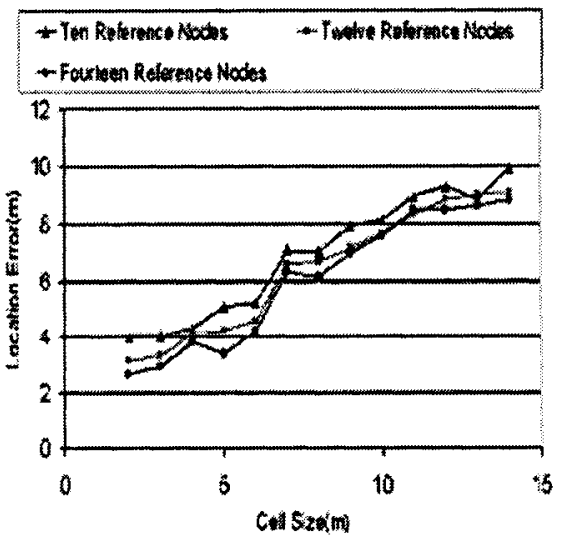

Fig. 10. Location Error against Cell size under varying number of Reference Nodes 
Fig. 9 shows that, the location error decreases with the increase in number of reference nodes. As the total number of refernce nodes increases, the number of reference nodes that are in the range of user node also increases which results in the decrease in location error. Fig. 10 shows the effect of cell size on location accuracy when the number of reference nodes are ten, twelve and fourteen. It shows that, the smaller the cell size, the better the accuracy of the system irrespective of the number of reference nodes. This decrease in location error is attributed to the increase in the number of points where signal strength vectors are estimated and compared with the received signal strength vectors. Therefore large cell size has to be chosen for coarsegrain requirement and small cell size for fine-grain requirements.

\section{Conclusions}

In this paper we have proposed a technique called TAIL which works in two levels using both range-free and range-based techniques in a sequence. TAIL uses the range-free technique to find the Locality of user node and then use multi-wall propagation model inside this Locality to find out the exact position. TAIL provides improved accuracy because we are using multi-wall propagation model which corrects the attenuation caused by obstructions in the building. We have shown through experiments that the error obtained is less than two meters, which is appreciable; and processing required is less than the methods present hitherto, as the exact location is estimated from the Locality identified by the Macro-level part of the TAIL as opposed to considering the whole operating environment.

\section{References}

1. Nissanka B. Priyantha, Anit Chakraborty and Hari Balakrishnan, The Cricket LocationSupport system, Proc. 6th ACM MOBICOM, August 2000.

2. R. Want, A. Hopper, V. Falcao, and J. Gibbons, The active badge location system, ACM Transactions on Information Systems, vol. 10, pp. 91--102, Jan. 1992

3. D. Niculescu and B. Nath, Ad hoc positioning system (APS) using AoA, In Proceedings of IEEE INFOCOM 2003, pp 1734-1743, April 2003.

4. P. Bahl and V. N. Padmanabhan, RADAR: An in-building RF-based user location and tracking system, In proceedings of the IEEE INFOCOM, pp 775-784, March 2000.

5. Loukas Lazos and Radha Poovendran, SeRLoc: Robust Localization for Wireless Sensor Networks, ACM Transactions on Sensor Networks, Vol. 1, pp 73-100, August 2005.

6. D. Niculescu and Badrinath, DV Based Positioning in Ad Hoc Networks, In Journal of Telecommunication Systems, 2003.

7. Nirupama Bulusu, John Heidemann, and Deborah Estrin, GPS-less Low-Cost Outdoor Localization for Very Small Devices, IEEE Personal Communications, October 2000

8. T. S. Rapport, Wireless Communications - Principles and Practice, IEEE Press 1996

9. Tian He, Chengdu Huang, Brian M. Blum, John A. Stankovic and Tarek Abdelzaher, Range-Free Localization Schemes for Large Scale Sensor Networks, In Proceedings of MobiCom '03, September 2003.

10. P. Enge and P. Misra, Special Issue on GPS: The Global Positioning Systems, Proceedings of the IEEE, January 1999. 